\title{
Hawk Eye: \\ A Logical Innovative Technology Use in Sports for Effective Decision Making
}

\author{
Baljinder SINGH BAL* • Gaurav DUREJA**
}

The present study aims at explaining hawk eye technology in sports with regard to their latest trends in the sporting arena. This is one of the most commonly used technologies in the various sports. It has been put to a variety of uses, such as providing a way to collect interesting statistics, construct very suggestive visual representations of the game play and even helping viewers to better understand the umpiring decisions. The Hawk-eye is one such technology which is considered to be really top indentation in various sports. The necessary idea is to monitor the trajectory of the ball during the entire duration of play. We will see how the Hawk-eye technology successfully treats each of these issues and provides a robust system to be used in practice. The Hawk-eye system was developed as a replay system, originally for TV Broadcast coverage. We have thus seen that the Hawk-eye is a great innovation, which puts technology to good use in the field of sports. The international scenario of hawk eye has been discussed while analyzing the data of hawk eye software for reliable decision. The technology is used widely these days, in sports such as Tennis and Cricket. The accuracy which can be achieved with the use of the system is making the authorities think seriously about reducing the human error component involved in important decisions. Hawk eye (artificial judgement) is very excellent and superb decision making power as compared to human being (natural judgement). Hawk-Eye software team is already working on the implementing system for basketball, football, badminton and snooker.

Keywords: Hawk-eye, Technology, cricket, tennis, football, snooker

* Department of Physical Education (T), Guru Nanak Dev University, Amritsar, Punjab India

** Department of Physical Education (TE\&L), Post Graduate Government College, Sector-11, Chandigarh (U.T), India 


\section{Introduction}

The Hawk-Eye Officiating System is the first and only ball-tracking system to have passed stringent ITF testing measures. It is accurate, reliable and practical. Hawk-Eye is one such technology which is considered to be really top notch in various sports. Hawk-Eye offers a unique blend of innovation, experience and accuracy that has revolutionised the sporting world. The basic idea is to monitor the trajectory of the ball during the entire duration of play. This data is then processed to produce life like visualizations showing the paths which the ball took. Such data has been used for various purposes, popular uses including the LBW decision making software and colourful wagon wheels showing various statistics in cricket. This paper attempts to explain the intricate details of the technology which goes behind the Hawk-Eye.

"In my 20 years in professional tennis, this is one of the most exciting things to happen for players, fans and television viewers. This new technology will add a whole new dimension to the game." - Andre Agassi -

We first start off with a general overview of the system and an outline of the challenges that we might face, then move on to the details of the technology and end with various applications where one sees this technology being put to use. Hawk-Eye technology used in sports programming is constantly evolving, with specialist cameras becoming more advanced and working methods speeded up through technological advancements.

Innovations such as Hawk-Eye are for the betterment of sports. First used in cricket and later adopted by various sports such as tennis and snooker, have been revolutionary in sports TV. It's at big events such as the Olympics that technologies similar to this are brought to the attention of makers. Hawk-Eye captures and processes images from a number of cameras to track the path of a ball in three dimensions on an imaginary cricket pitch. Hawk-Eye uses output from the existing broadcast television cameras rather than utilizing special cameras of its own with higher frame rates (Bijker, Hughes, \& Pinch, 1987). Hawk-Eye is a multifarious computer system used in cricket, tennis and other sports to visually track the path of the ball and display a record of its most statistically likely path as a moving image. In some sports, like tennis, it is now part of the intercession process. It is also used in some instances to predict the future path of a ball in cricket. It was developed by engineers at Roke Manor Research Limited of Romsey, Hampshire in the UK, in 2001. A UK patent was submitted but withdrawn by Dr Paul Hawkins and David Sherry. Later, the technology was spun off into a separate company, Hawk-Eye Innovations Ltd., as a joint venture with television production company Sunset with Vine. Through 
hawk eye technology people attract more towards technology and interested in viewing sporting events and also developed the mental level of spectators.

"As a player, and now as a TV commentator, I always dreamed of the day when technology would take the accuracy of line calling to the next level. That day has now arrived." - Pam Shriver -

Sport is an ever-green field and attracts big spending each year. The requirement and expectation of users have grown significantly since digital media is popular (Wang \& Parameswaran, 2004). Hawk eye is basically is the combination of sports and engineering background brain with manipulate the things and create illusion in the field of sports. This type of excellent technology provide to us in the new world of graphics to view the crucial decision in very easy manner. Use the size of goal mouth to determine the size of soccer ball in their ball tracking process. If we think about the last 20 or 30 years we all depends only the human judgment either is right or wrong but present time directly challenges the human judgment with the hawk eye technology. At present scenario we can say that our minute to minute reaction in any sports directly calculate the things through this technology. There are numbers of cameras which cover the micro seconds of movements of the players so that capture the right decision for the game. Hawk eye technology captured the cricket balls, tennis balls; soccer ball, snooker balls and movement of players and in the future it cover badminton and basketball etc. There is also an attempt to automatic detect off-side situation in soccer (Lam et al., 2003). The present manifests discuss about various games in which hawk apply or is going to plan the implement this futuristic technology.

\section{Hawk-Eye Use in Various Sport}

Cricket. Hawk eye technology is implementing in cricket with lot of colour graphics for their perfect view of every ball. Hawk-Eye technology as an additional coaching and training tool and is only the fourth such installation currently is being deployed in the cricketing world. Hawk eye technology was first used by Channel 4 during a Test match between England and Pakistan on Lord's Cricket Ground, on 21 May 2001. It is used primarily by the majority of television networks to track the trajectory of balls in flight. In the winter season of 2008/2009 the ICC trialled a referral system where, Hawkeye was used for referring decisions to the third umpire if a team disagreed with an LBW decision. The third umpire was able to look at what the ball actually did up to the point when it hit the batsman, but could not look at the predicted flight of the ball after it hit the batsman. Its major use in cricket broadcasting is in analysing leg before wicket decisions, where the likely path of the ball can be projected forward, through the batsman's legs, to see if it would have hit the stumps. Consultation 
of the third umpire, for conventional slow motion or Hawk-Eye, on leg before wicket decisions, is not currently sanctioned in international cricket and doubts remain about its accuracy in cricket. Due to its real time coverage of bowling speed, the systems are also used to show delivery patterns of bowler's behaviour such as line and length, or swing/turn information. At the end of an over, all six deliveries are often shown concurrently to show a bowler's variations, such as slower deliveries, bouncers and leg-cutters. A complete record of a bowler can also be shown over the course of a match. Batsmen also benefit from the analysis of Hawk-Eye, as a record can be brought up of the deliveries batsmen scored from. These are often shown as a 2-D silhouetted figure of a batsmen and colour-coded dots of the balls faced by the batsman. In sequence such as the exact spot where the ball pitches or speed of the ball from the bowler's hand (to gauge batsman reaction time) can also help in post-match analysis.

Figure 1. Pinpoint the Line, Length, Speeds, Swing \& Seam movement of each delivery bowled in hawk eye.

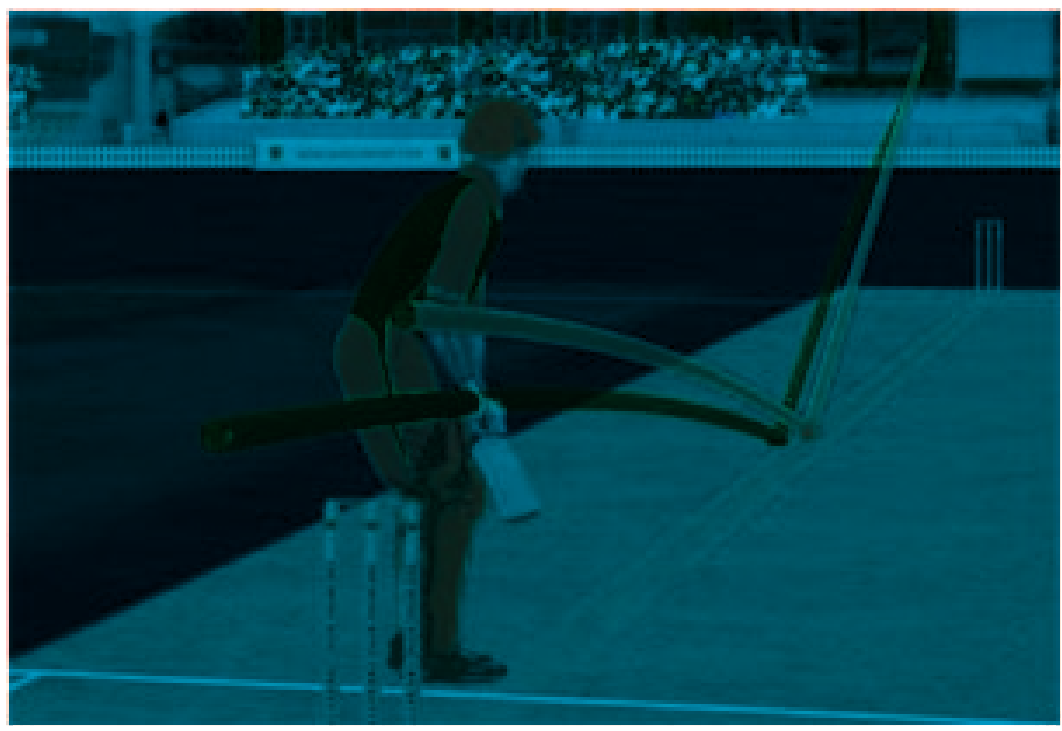

Source: www.hkcc.org/section8_1_18.html

In cricket, hawk eye technology use in manly lbw decision at the crucial point against the umpire decision. This technology gives $100 \%$ of their critical decision view to the third umpire for correct decision. But some of the people criticism about this technology because the reason is that this type of technology 
is more edge over the human being. But if we are looking overall about hawk eye technology this is going very depth in cricket with lot of variations like, wagon wheels, pitch maps, Beehives, rail cam, ball speeds and reaction of the catch. At present cricket arena every batsman, bowler and fielder records their match practice movement with hawk eye technology to improve their playing styles.

Figure 2. Hawk-Eye analysis of bowling in cricket

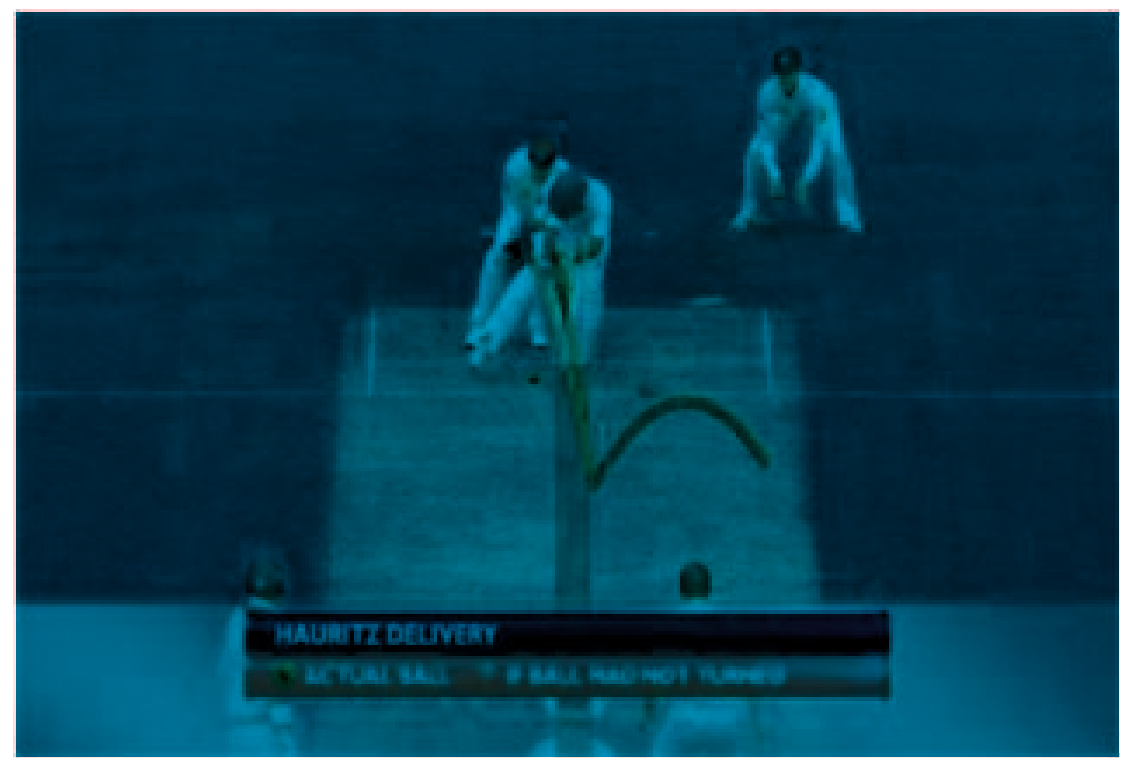

Source: http://singularityhub.com/wp-content/uploads

Tennis. It is an Olympic event and playing with very enthusiasm. The professional game is won and lost on the smallest of margins. Players leave no margin for error in their practice, preparation or play. Why should line calling be any different? Hawk-Eye was tested by the International Tennis Federation (ITF) in New York City and was passed for professional use. Hawk-Eye reported that the New York tests involved 80 shots being measured by the ITF's high speed camera, a device similar to Mac-Cam. During an early test of the system during an exhibition tennis tournament in Australia (seen on local TV), there was an instance when the tennis ball was shown as "Out", but the accompanying word was "In". This was explained to be an error in the way the tennis ball was shown on the graphical display as a circle, rather than as an ellipse. This was immediately corrected. Hawk-Eye has been used in television coverage of 
several major tennis tournaments, including Wimbledon, the Stella Artois at Queens, the Australian Open, the Davis Cup and the Tennis Masters Cup. The US Open Tennis Championship announced they would make official use of the technology for the 2006 US Open where each player receives two challenges per set. It is also used as part of a larger tennis simulation implemented by IBM called Point Tracker.

Figure 3. Hawk-Eye system is widely used in tennis

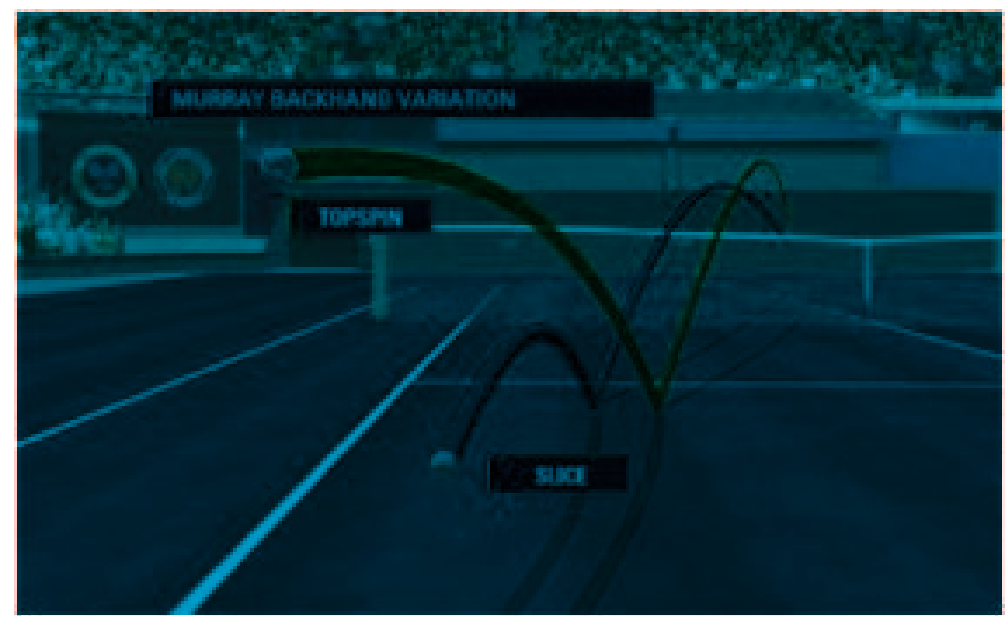

Source: http://i.dailymail.co.uk/i/pix

"As a player, I want to know the line calls are as accurate as technology will allow. In that sense, (Hawk Eye) is great news for all players." - Maria Sharapova -

The 2006 Hopman Cup in Perth, Western Australia, was the first elite-level tennis tournament where players were allowed to challenge point-ending line calls, which were then reviewed by the referees using Hawk-Eye technology. It used 10 cameras feeding information about ball position to the computers. In tennis, lots of decision depends on the line because maximum time ball is on the line or just touch the edge of the line this hawk eye technology helps the tennis players with perfect decision with slow motion replay. In tennis scenario this is one of the perfect and best video analysis software for increase the standards of tennis championship and players performance. 
Figure 4. Uses an array of cameras that feed three dimensional coordinates of the flight of the tennis ball

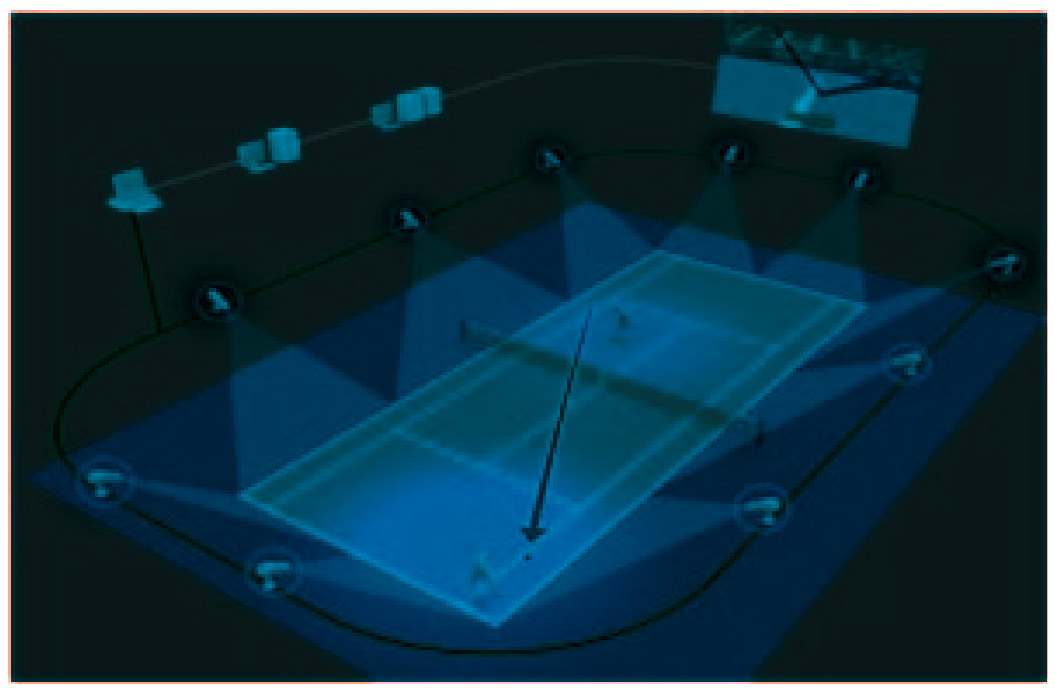

Source: http://www.studiosayers.com/storage/post-images/hawkeye_blog

Snooker. It is playing on the table with one stick and lots of ball. Hawk eye technology team start their micro movement with snooker. BBC used HawkEye in World Snooker Championship in 2007 for the first time in its television coverage to show player views, particularly in the incidents of potential snookers. It has also been used to demonstrate intended shots by players when the actual shot has gone awry. It is now used by the BBC at every World Championship, as well as some other major tournaments. The BBC uses the system sporadically, for instance in the 2009 Masters at Wembley the Hawkeye was at most used once or twice per frame. In contrast to tennis, the Hawk-Eye is never used in snooker to assist referees' decisions. 
Hawk Eye

Figure 5. Animated shots of snooker in hawk eye system

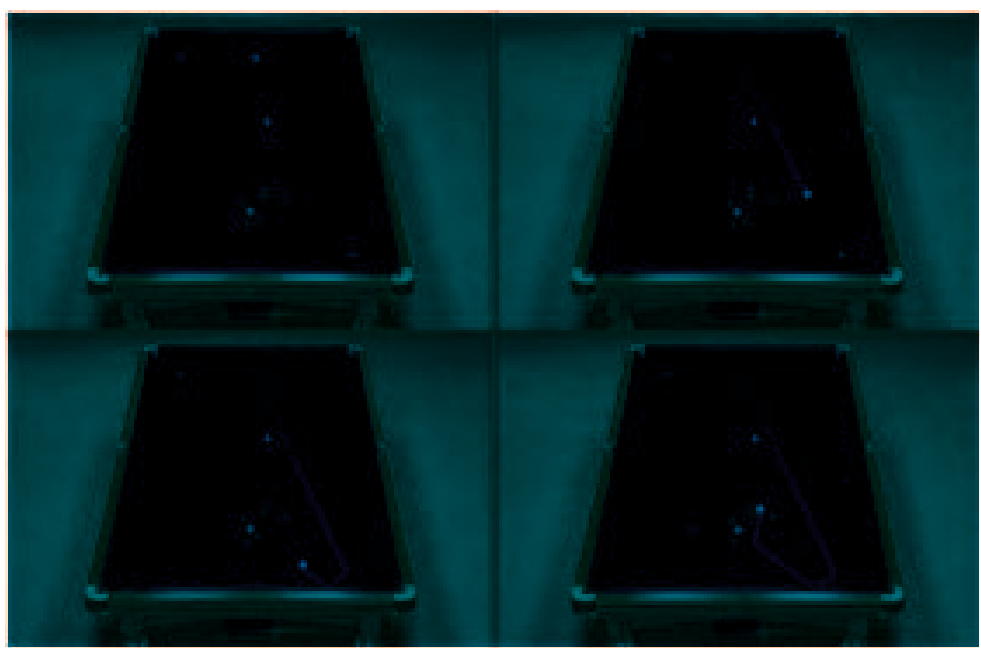

Source: http://singularityhub.com/wp-content/uploads

Football. It is the most popular game in the world. Physically and mentally football is known as tough game and famous for very stiff competitions. For the popularity of football game there is desire need of hawk eye technology in the goal line of the football field. Football is the only on e of the games that have lots fan as compared to other games. Paul Hawkins the man who invented the technology said we would install it free of charge in every Premier League ground if we could have the rights to sell the sponsorship. The cost of installation of the patented system would be between $£_{100,000}$ and $£_{1} 125,000$ per stadium. "That just highlights that it is commercially practicable and that the cost would not be an issue. "For example in tennis Rolex pays to the All England Club a lot more than the All England Club pays to us." FIFA president Sepp Blatter has previously dismissed the possibility of using goal-line technology in football, but in the wake of controversies at the World Cup he has promised to reconsider the feasibility of such a system. Hawk-Eye is a computer system currently used in sports including cricket and tennis where the path of the ball is visually tracked to display a record of its most statistically likely path as a moving image. 
Figure 6. Hawk-Eye would involve cameras around each goalmouth

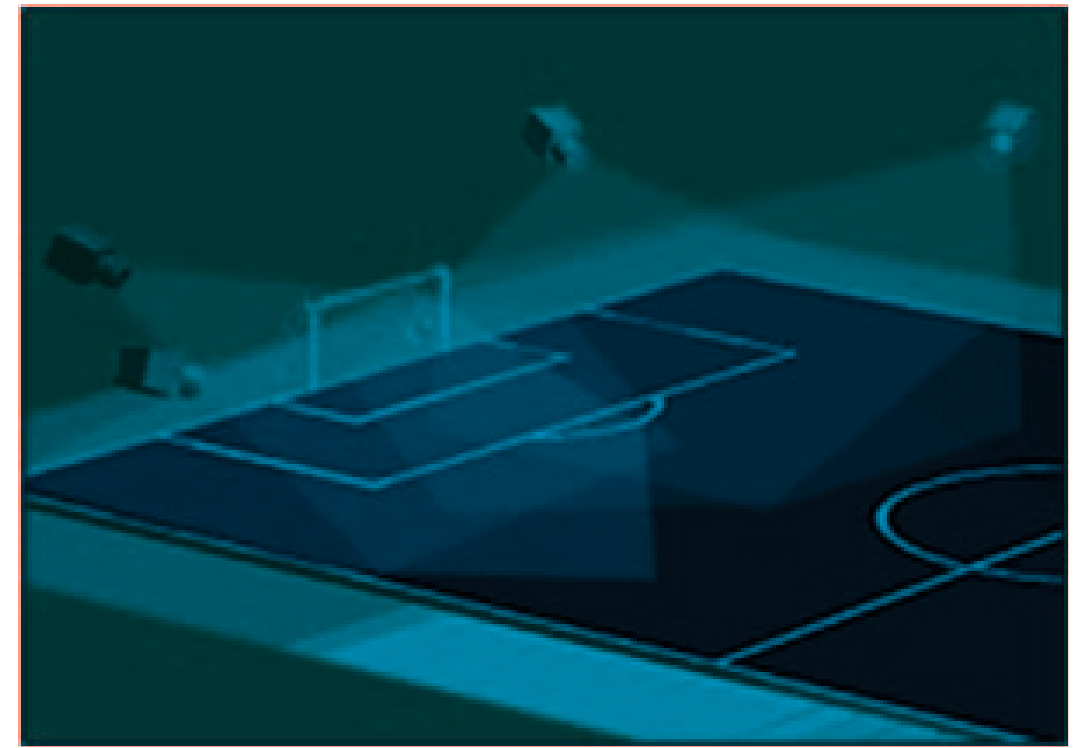

Source: http://newsimg.bbc.co.uk/media/images/44003000/gif

Badminton. It is an Olympic event and world's fastest racquet sport and at the moment does not utilise any sort of technology to assist with umpire decisions. The fast pace of the games and the intense speed with which the shuttlecock lands on the ground, can sometimes make it tricky for umpires to judge whether a point was scored or not. A lot of players, including the world number one Lee Chong Wei, have called for a technology to be introduced into the game similar to what is used in tennis. It does not look like any sort of technology will be introduced into the sport anytime soon but the organising committee of the sport will look into the matter again after the 2012 Olympics. In badminton or any kind of game you have to react decision in micro seconds. Whatever happens, badminton as a game will not change; it will simply evolve along with the times to come. Technology is all around us and it is high time that the world's biggest sports stand up and follow the rest of the crowd. Hawk eye team is developed the software for better results in badminton.

\section{Reaction Time}

The amount of time required to respond to a stimulus. Typically, stimuli are either visual or auditory. To measure the reaction of the players during catch in match, backhand and forehand to hit the tennis ball, kick the football, hit in 
snooker and lots of other things for reaction time; this is one of the vital things to enhance the human being agility. A Hawk-Eye Reaction Time is a simple yet valuable tool for demonstrating how quickly a particular bowler is pitching. The graphic can also be applied to a catch, thus quantifying a spectacular replay or slow-motion shot.

Figure 7. Reaction time check during catch

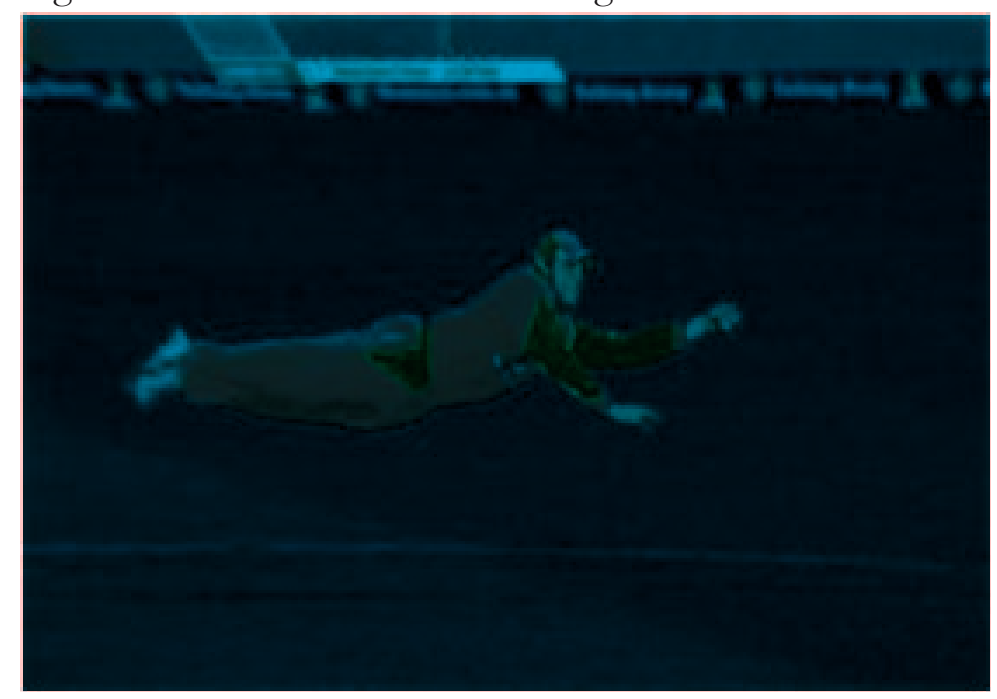

Source: http://www.hawkeyeinnovations.co.uk/?page_id=1012

\section{Discussion}

In this paper, an attempt has been made to provide the latest knowledge of hawk eye technology in various games. On this high definition of coloured graphics with great quality of slow motion replay they scan the whole body of the players. In this entire manifest we discuss about the qualities which give benefit to players for enhance their games. Now we discuss about artificial judgement (Hawk Eye) and natural judgement (Human Being). At present scenario artificial judgment is edge over the human judgment. That means hawk eye technology is better and excellent decision making power as compared to human being judgement. Because in present sporting arena maximum decision is depends on the final decision of the hawk eye analysis software. If hawk eye technology display yes or no it will be count full a final decision against the players. So there are lots of advantage and lots disadvantages of hawk eye 
technology. Although Hawkeye is very accurate in measuring the actual path of a ball, when it comes to predicting the future path of the ball, such as in LBW decisions, it is not as clear. If the ball is heading to the pitch, there's no way Hawk-eye can tell if a delivery is going to skid a bit more than normal or hit a crack, bit of grass, or worn patch of the pitch. The predicted path of the ball is based on the average and expected pathway. There are lots work in this area includes the hawk technology for better future (Rui, Gupta \& Acero, 2000; Nepal, Srinivasan \& Reynolds, 2001; Yow, Yeo, Yeung \& Liu 1995). We are going towards a technology world with every field required some kinds of technology for better results. This type of technology gives the player to remove their minor or major error in their playing style and create image in future for better results in the record books. As per the decision concerned about the hawk eye technology that they also no $100 \%$ accurate tell the position but the entire frame they create the trajectory of the ball in which they finally take position that's why we all accept the things that this might be right decision as compared to our decision. The first umpire is saying he is skilled at knowing how things are while the second umpire is implying that his knowledge of what happens is worthy of respect (Collins, 2010). Both umpires are claiming a degree of epistemological privilege. There is one most important point in hawk eye technology that they never tired but human being tired at some point for crucial decision. Russell analyses the role of umpires into a performative aspect and an observational aspect using the language of the philosopher Austin but he is not concerned with the tension between them except as a problem for providing a coherent philosophical account of the umpire's job (Yu et al., 2003). There is a danger that Hawk-Eye as used could inadvertently cause naïve viewers to overestimate the ability of technological devices to resolve disagreement among humans because measurement errors are not made salient (Collins \& Evans, 2008). There los of material provided regarding this technology an article in Scientific American (Fischetti, 2007) and a recently published analysis of line calls in tennis (Mather, 2008). The sports fraternity is big fans of hawk eye technology software for the fair decision and also makes interesting view of point of different player's style.

\section{Conclusions}

At international level hawk eye is the part and parcel of competitive sports at present. Hawk-Eye is a great innovation, which puts technology to good use in the field of sports. The technology is used widely these days, in sports such as Tennis and Cricket. At present Hawk-Eye technology only implement in those sports which do not have duration of play. If hawk eye technology implement in the duration games then interest of the games is less. Hawk eye software is an excellent instrument which can be used by players, mangers, statisticians, 
tacticians, coaches to analyze previous games and come up with new strategies for better results. At present scenario hawk eye technology software is one of the vital components for decision in sports.

\section{References}

Bijker, W. E., Hughes, T. P., \& Pinch, T. J. (1987). The Social Construction of Tech nological Systems: New Directions in the Sociology and History of Technology. Cambridge, MA and London: MIT Press.

Collins, H., \& Evans, R. (2008). You cannot be serious! Public understanding of technology with special reference to "Hawk-Eye". Public Understanding of Science, 17(3), 283-308.

Collins, H.M. (2010). Tacit and Explicit Knowledge. Chicago: The University of Chicago Press.

Fischetti, M. (2007). "In or Out? Scientific American, July, 96-97.

Lam, M., Chan, M., Leung, J., Wong, R., Hang, C. C., \& Jin, J. S. (2003). Computerassisted off-side detection in soccer matches, Technical Report. School of Information Technologies, University of Sydney.

Mather, G. (2008). Perceptual Uncertainty and Line-Call Challenges in Professional Ten nis. Proceedings of the Royal Society B 10.1098/rspb.2008.0211

Nepal, S., Srinivasan, U., \& Reynolds, G. (2001). Automatic detection of 'Goal' seg ments in basketball videos. Proc. ACM MM'01, Ottawa, 261-269.

Rui, Y., Gupta, A., \& Acero, A. (2000). Automatically extracting highlights for TV Baseball programs. Proc. of ACM Multimedia'00, 105-115.

Russell, J. S. (1997). The Concept of a Call in Baseball. Journal of the Philosophy of Sport, XXIV, 21-37.

Wang, J. R., \& Parameswaran, N. (2004). Survey of Sports Video Analysis: Research Issues and Applications. Paper presented in Conferences in Research and Practice in Information Technology, Sydney.

Yow, D., Yeo, B. L., Yeung, M., \& Liu, B (1995). Analysis and presentation of soccer highlights from digital video. Proc. ACCV'95. 
Yu, X., Xu, C. S., Leong, H. W., Tian, Q., Tang, Q., \& Wan, K. W. (2003). Trajectorybased ball detection and tracking with applications to semantic analysis of broadcast soccer video. Proc. of ACM MM'03, Berkeley, 11-20.

Baljinder SINGH BAL, Ph.D., is working as Assistant Professor in the Department of Physical Education (T) at Guru Nanak Dev University Amritsar, Punjab, India. He received the B.P.E., M.P.E. and M.Phil degree in Physical Education from Lakshmibai National Institute of Physical Education (Deemed University) Gwalior (M.P.) India. He obtained his Ph.D. from Panjab University, Chandigarh under the esteemed supervision of Late. Prof. (Dr.) Ajmer Singh, (Olympian) Arjuna Awardee. Dr. Baljinder Singh Bal is the recipient of 1st JSHR Research Award 2009 (Martos) Spain. His areas of interest include Biomechanics and Motor Control of Human Movements, Science of Sports Training and Hatha Yoga Pradipika.

Gaurav Dureja, Ph.D., is P.G.D.C.A, B.P.Ed and M.P.Ed from Guru Nanak Dev University, Amritsar. He did his M.Phil in Physical Education from Panjab University, Chandigarh and is currently pursuing Ph.D. in Physical Education from same University. A gold medalist in senior national and inter-varsity softball championships, he is working as Assistant Professor for postgraduate studies in the department of Physical Education (TE\&L) at Post Graduate Government College, sector 11, Chandigarh since 19th August 2010. His area of interest is Use of Information Technology in Physical Education and Adapted Physical Education. 